\title{
Experimental Investigation of Pitch Ramp-Hold-Return Motion of Flat Plates at Low Reynolds Number
}

\author{
Huai-Te $\mathrm{Yu}^{1}$, Luis P. Bernal ${ }^{2}$ and Chris Morrison ${ }^{3}$ \\ University of Michigan, Ann Arbor, MI, 48109
}

The flow development and force generation of a two-dimensional flat plate undergoing a pitch ramp-hold-return motion are reported. Direct force measurements and PIV flow field measurements are described. The experiments were conducted in water at a Reynolds number based on free stream speed and chord of approximately 5,000 and reduced frequency $k=0.2$, where the reduced frequency is based on the rate of change of the pitch angle normalized with chord and free stream speed. The maximum pitch angle and the duration of the hold part of the motion were varied and their impact on the force generation and flow evolution were measured. As expected the force and flow evolution during the pitch ramp part of the motion is independent of the hold and return parts and is in agreement with previous work reported in the literature. It is found that the evolution of aerodynamic parameters during the hold part is a function of time and maximum pitch angle but independent of hold duration. However the force and flow evolution during the return part depends on the hold duration and maximum pitch angle. PIV measurements are used to document the evolution of the LEV and TEV vortices.

\section{Nomenclature}

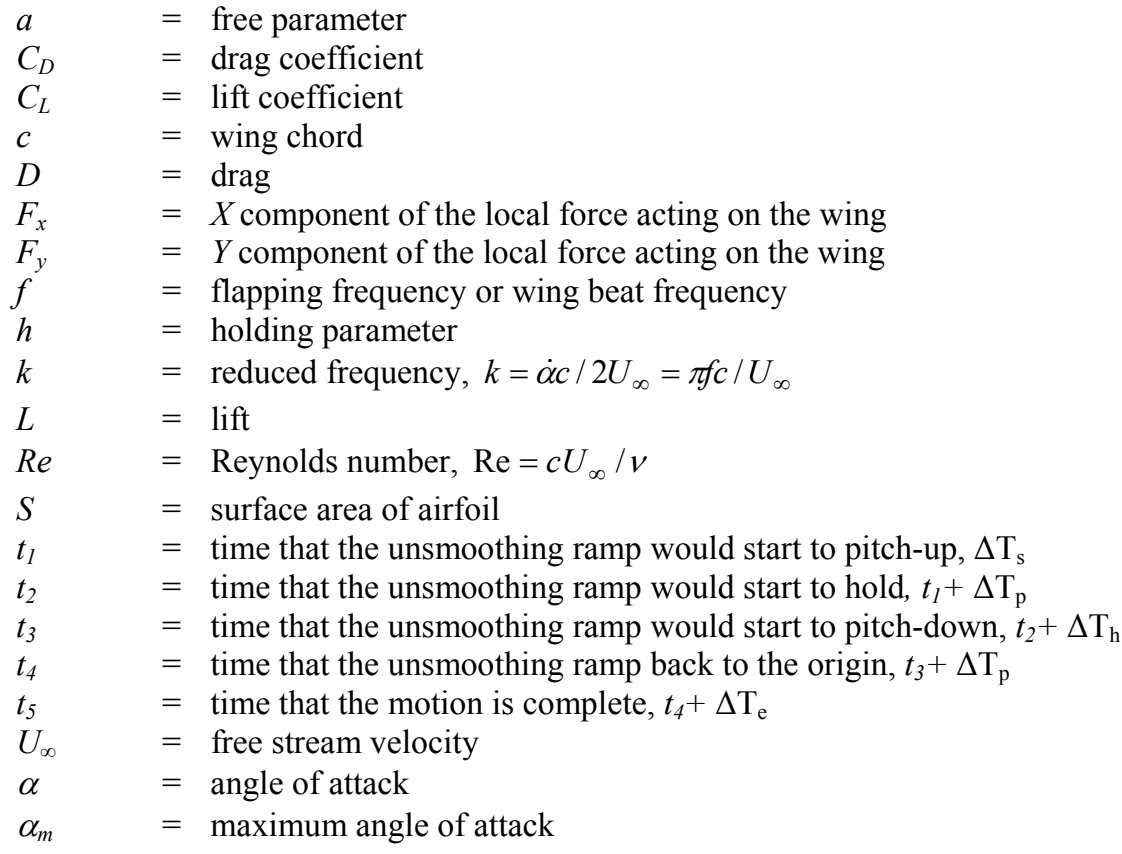

${ }^{1} \mathrm{PhD}$ student, Department of Aerospace Engineering, University of Michigan, htyu@umich.edu, AIAA Student Member.

${ }^{2}$ Associate Professor, University of Michigan, Department of Aerospace Engineering, 1pb@umich.edu, AIAA Member.

${ }^{3} \mathrm{PhD}$ student, Department of Aerospace Engineering, University of Michigan, cmorrisz@umich.edu, AIAA Student Member. 


$\begin{array}{ll}\Delta \mathrm{T}_{\mathrm{p}} & =\text { pitching duration } \\ \Delta \mathrm{T}_{\mathrm{s}} & =\text { starting duration } \\ \Delta \mathrm{T}_{\mathrm{h}} & =\text { holding duration } \\ \Delta \mathrm{T}_{\mathrm{e}} & =\text { ending duration } \\ v & =\text { kinetic viscosity of water }\end{array}$

\section{Introduction}

$\mathrm{T}$ HIS paper presents the results of an investigation of the unsteady aerodynamics of a flat plate undergoing the linear pitch ramp, hold and return kinematics proposed by the AIAA Fluid Dynamics Technical Committee (FDTC) Low Reynolds Number Discussion Group (LRDG) ${ }^{1}$. This motion kinematics is relevant to the study of the aerodynamics of aggressive perching maneuvers where a rapid increase in angle of attack beyond quasi steady stall could be used to produce large lift and drag forces. Ol et al provides an overview of the main features of the flow. Large LEV vortices are formed during the pitch-up part of the motion and detaches as the flow evolves in time. It is found that at moderate values of the reduced frequency noncirculatory (i.e. inertia) effects are important and cause an increase in lift well above the quasi steady value for leading edge pivot axis. It is also found that motion history effects are important for the return part of the motion.

In a related study Granlund et al. ${ }^{2}$ consider the aerodynamics of a pitch-up maneuver and extended prior results to a maximum pitch angle of 90 degrees. They found that pivot point location and reduced frequency play an important role on aerodynamic loads. Pitching about the leading edge produces significantly higher lift in the initial acceleration part of the motion due to noncirculatory effects even at low reduced frequencies. Similar motion kinematics was investigated by Yeon et al. ${ }^{3}$. They derived the aerodynamic forces from the PIV data instead of direct measurement. The PIV data was also used to determine the LEV and TEV vortex evolution. At moderate reduced frequency $(\mathrm{k}=0.2)$ a strong LEV vortex forms early in the motion followed by a relatively weak TEV. However, at high reduced $(\mathrm{k}=1)$ frequency the TEV forms before the LEV.

$\mathrm{Ol} \mathrm{et} \mathrm{al.}{ }^{4}$ also considered the pitch return part of the motion and found that during the upstroke the lift coefficient normalized by reduced frequency is a simple function of the rate of change of the angle of attack. During the downstroke flow exhibits history effects from preceding parts of the motion, which invalidate the simple reduced frequency scaling. Other contributions using computational techniques have been reported by Eldredge et al ${ }^{5}$, Garmann and Visbal ${ }^{6}$, and Lian and $\mathrm{Ol}^{7}$.

The current study is continuation of prior work of the pitching ramp-hold-return kinematics. We investigate the effects of the duration of the holding part of the motion at several maximum angular amplitudes. The experiments are conducted at a Reynolds number approximately equal to 5,000 with reduced frequency of 0.2 in a water tunnel, and report force and PIV measurements.

\section{Experimental Setup}

\section{A. Kinematics}

The pitch ramp-hold-return motion was produced by a stepping motor (Model RK266-03A-P1), a rotary stage (Velmex B4872TS Rotary Table) and the associated computer control system. The pitch angle history is a trapezoid with the corners smoothed using a slightly modified Eldredge's function, as shown in Eq (1). The motion time history is shown at the left hand size of Figure 1, where the prefix of $t$ s represents for smoothing transient. The parameter $a$ in Eldredge's function was set to 11, which provides approximately 4.5 degrees transition at the trapezoid corners. One purpose of this smoothing region is to avoid model vibration in the experiments; motion acceleration is confined to these narrow regions of time. For the present implementation of the kinematics a discretization time step of 0.04 seconds was used which corresponds to $0.08^{\circ}$ increments for $\mathrm{k}=0.2$. The actual motion implementation is illustrated in Figure 1 where individual stepper motor commands are shown as circles. The free stream velocity used in this study is $6.662 \mathrm{~cm} / \mathrm{s}$.

$$
\alpha(t)=\frac{k}{a} \ln \left[\frac{\cosh \left(a U_{\infty}\left(t-t_{1}\right) / c\right) \cosh \left(a U_{\infty}\left(t-t_{4}\right) / c\right)}{\cosh \left(a U_{\infty}\left(t-t_{2}\right) / c\right) \cosh \left(a U_{\infty}\left(t-t_{3}\right) / c\right)}\right]
$$




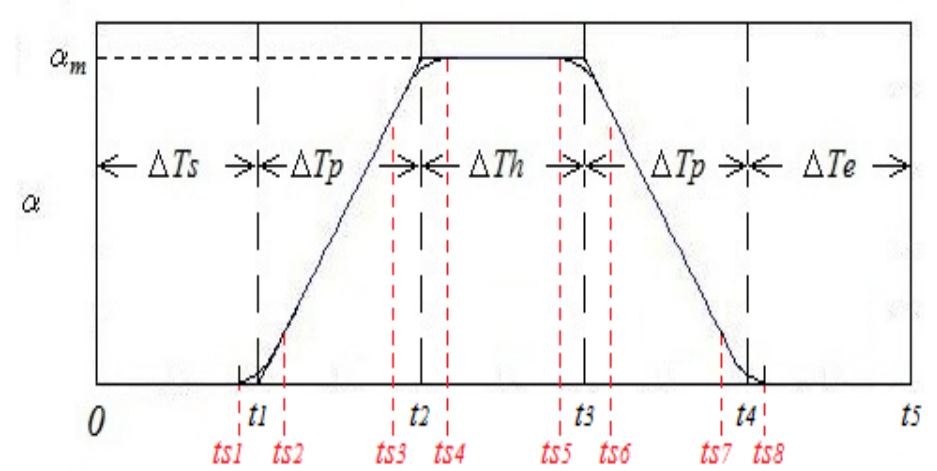

(a)

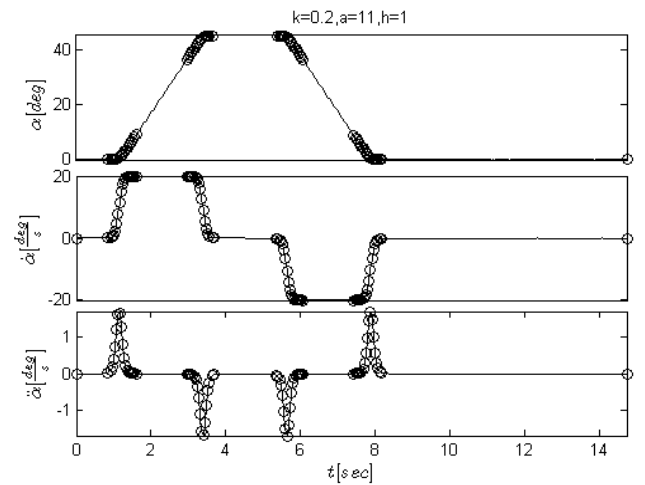

(b)

Figure 1. Schematics of (a) pitch ramp-hold-return motion and (b) the VELMEX implementation

\section{B. Force Measurement}

A Mini40 F/T transducer manufactured by ATI Industrial Automation with SI-80-4 calibration was used to measure forces. The wing model is attached to the metric side of the sensor using a bracket and the fixed side of the sensor is attached to the rotary stage. The wing model is mounted vertically in water tunnel as shown in Figure 2(a). The coordinates systems used are shown in Figure 2(b). The force sensor measures force normal to the model chord, $\mathrm{F}_{\mathrm{y}}$, along the chord, $\mathrm{F}_{\mathrm{x}}$, and along the model span, $\mathrm{F}_{\mathrm{z}}$. Force data processing is performed in the sensor frame of reference and then converted to Lift and Drag as shown in Figure 2 (b).

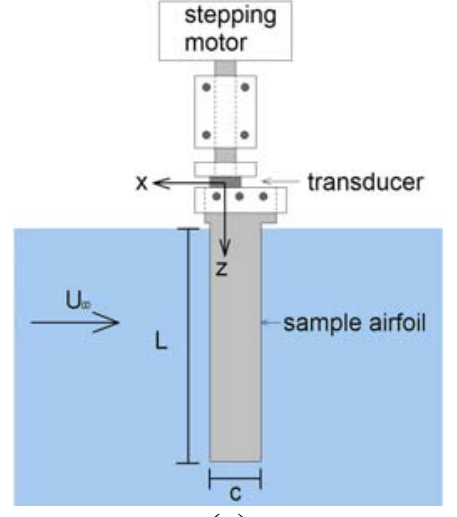

(a)

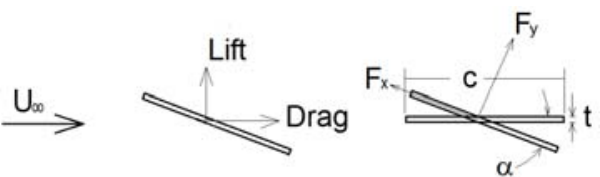

$$
\begin{aligned}
& L=F_{y} \cos \alpha+F_{x} \sin \alpha \\
& C_{L}=\frac{L}{\left(\rho U_{\infty}^{2} S\right) / 2}
\end{aligned}
$$

(b)

Figure 2. Schematics of (a) force measurement system; (b) airfoil coordinate system. (draw not in scale)

We considered three maximum angular amplitudes, $33^{\circ}, 45^{\circ}$, and $57^{\circ}$; for which three different holding parameters values were considered $0.5,1$, and 2 . The holding parameter is defined as the holding duration normalized by the pitch ramp duration, which varies with maximum angular amplitude. The test cases are summarized in Table 1. A flat plate with 3" chord is used with $6.25 \%$ thickness to chord ratio and rounded leading and trailing edges. The model is made of stainless steel and polished to minimize surface glare in the PIV measurements. For the present experiments the model span immersed in water is 23 " and the distance from the wing tip to the bottom glass wall is less than $5 \mathrm{~mm}$.

Force/torque data were acquired using a DAQ Card, NI USB 6225 at a sample rate of $1000 \mathrm{~Hz}$, and the data were transferred to a computer for further processing. A 6 order zero-phase Butterworth low-pass filter with cutoff frequency of $2 \mathrm{~Hz}$ was used to remove high frequency electronic noise and vibrational response of the sensor which is also at higher frequency than the hydrodynamic response times for each experiment. After the return motion a wait time of 6 convective time was added to each kinematic as shown in Table 1. This wait time was found sufficient for the flow to return to the initial state. For the present experiments the wing motion was repeated at least 49 times and the results are phase averaged. 
Table 1. Test matrix of force measurement

\begin{tabular}{|c|c|c|c|c|c|c|}
\hline $\mathrm{k}$ & $\alpha_{\mathrm{m}}$ & $\Delta \mathrm{T}_{\mathrm{s}}$ & $\begin{array}{c}\Delta \mathrm{T}_{\mathrm{p}} \\
\left(\mathrm{c} \alpha_{\mathrm{m}} / 2 \mathrm{k} \mathrm{U}_{\infty}\right)^{*} \\
\end{array}$ & \multicolumn{2}{|c|}{$\Delta \mathrm{T}_{\mathrm{h}}=\mathrm{h} \Delta \mathrm{T}_{\mathrm{p}}$} & $\Delta \mathrm{T}_{\mathrm{e}}$ \\
\hline \multirow{9}{*}{$\begin{array}{c}0.2 \\
(\mathrm{a}=11)\end{array}$} & \multirow{3}{*}{$33^{\circ}$} & \multirow{9}{*}{$\mathrm{c} / \mathrm{U}_{\infty}$} & \multirow{3}{*}{$1.44 \mathrm{c} / \mathrm{U}_{\infty}$} & $0.5 \Delta \mathrm{T}_{\mathrm{p}}$ & $\left(=0.72 \mathrm{c} / \mathrm{U}_{\infty}\right)$ & \multirow{9}{*}{$6 \mathrm{c} / \mathrm{U}_{\infty}$} \\
\hline & & & & $1 \Delta \mathrm{T}_{\mathrm{p}}$ & $\left(=1.44 \mathrm{c} / \mathrm{U}_{\infty}\right)$ & \\
\hline & & & & $2 \Delta \mathrm{T}_{\mathrm{p}}$ & $\left(=2.99 \mathrm{c} / \mathrm{U}_{\infty}\right)$ & \\
\hline & \multirow{3}{*}{$45^{\circ}$} & & \multirow{3}{*}{$1.96 \mathrm{c} / \mathrm{U}_{\infty}$} & $0.5 \Delta \mathrm{T}_{\mathrm{p}}$ & $\left(=0.98 \mathrm{c} / \mathrm{U}_{\infty}\right)$ & \\
\hline & & & & $1 \Delta \mathrm{T}_{\mathrm{p}}$ & $\left(=1.96 \mathrm{c} / \mathrm{U}_{\infty}\right)$ & \\
\hline & & & & $2 \Delta \mathrm{T}_{\mathrm{p}}$ & $\left(=3.93 \mathrm{c} / \mathrm{U}_{\infty}\right)$ & \\
\hline & \multirow{3}{*}{$57^{\circ}$} & & \multirow{3}{*}{$2.49 \mathrm{c} / \mathrm{U}_{\infty}$} & $0.5 \Delta \mathrm{T}_{\mathrm{p}}$ & $\left(=1.24 \mathrm{c} / \mathrm{U}_{\infty}\right)$ & \\
\hline & & & & $1 \Delta T_{p}$ & $\left(=2.49 \mathrm{c} / \mathrm{U}_{\infty}\right)$ & \\
\hline & & & & $2 \Delta \mathrm{T}_{\mathrm{p}}$ & $\left(=4.97 \mathrm{c} / \mathrm{U}_{\infty}\right)$ & \\
\hline
\end{tabular}

A tare procedure was developed to remove model inertia and model position effects on the hydrodynamic force measurements. Dynamic and static measurements in air and water were performed. Dynamic measurements are the measurements in air and water with non-zero free stream velocity, using the proper pitch ramp-hold-return kinematic. Static measurements are measurements in air and water with zero free stream velocity, in which the pitch angle is changed in increments of 5 degrees from $0^{\circ}$ to $90^{\circ}$ with 1 minute holding time for measurement. These data were used to remove the model inertia contribution and model angular position contribution to the measured force. A flow chart of the data processing is shown in Figure 3.

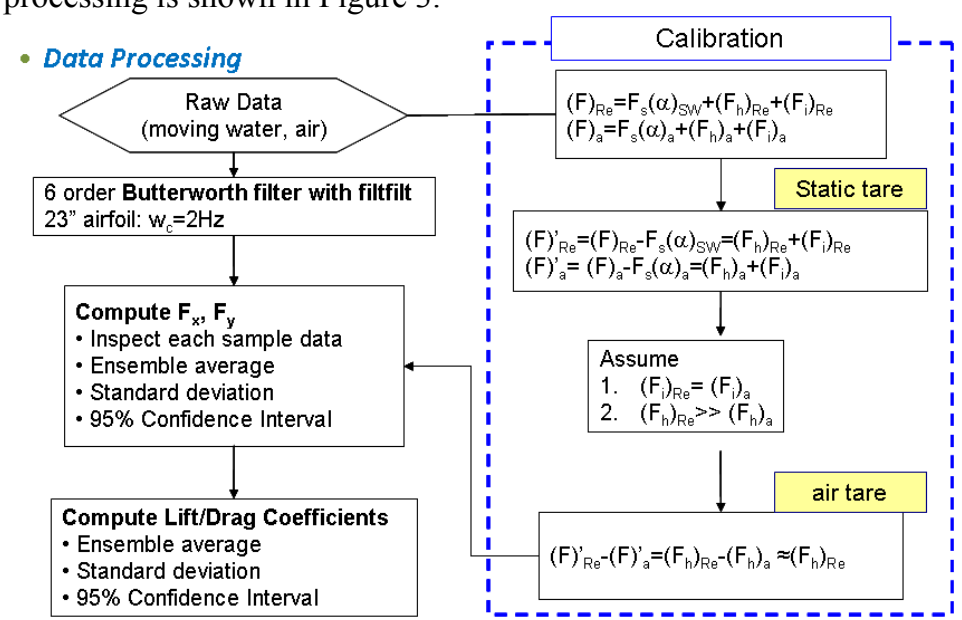

Figure 3. Flow chart of data processing, "Re" denotes the measurements in free stream velocity; "SW" denotes the measurements in the zero free stream velocity; "a” denotes the static measurement in air

An uncertainty analysis was conducted to determine the measurement uncertainty. The parameters of interest are the lift and drag coefficient, which were obtained from the independent variables, normal forces $\left(\mathrm{F}_{\mathrm{y}}\right)$ and axial force $\left(\mathrm{F}_{\mathrm{x}}\right)$, free stream velocity, wing chord (c) and span $(\mathrm{L})$. The accuracy for each independent variable, and denotes as " $w$ ". For forces, the $95 \%$ confidence intervals were used to characterize the accuracy, the accuracy for the wing chord and span were approximately $0.001 \mathrm{~m}$. For the free stream velocity, the accuracy was obtained from PIV measurement.

$$
w_{c_{L}}=\left\{\left(\frac{\partial C_{L}}{\partial F_{x}} w_{F_{x}}\right)^{2}+\left(\frac{\partial C_{L}}{\partial F_{y}} w_{F_{y}}\right)^{2}+\left(\frac{\partial C_{L}}{\partial U_{\infty}} w_{U_{\infty}}\right)^{2}+\left(\frac{\partial C_{L}}{\partial c} w_{c}\right)^{2}+\left(\frac{\partial C_{L}}{\partial L} w_{L}\right)^{2}\right\}^{1 / 2}
$$




$$
w_{c_{D}}=\left\{\left(\frac{\partial C_{D}}{\partial F_{x}} w_{F_{x}}\right)^{2}+\left(\frac{\partial C_{D}}{\partial F_{y}} w_{F_{y}}\right)^{2}+\left(\frac{\partial C_{D}}{\partial U_{\infty}} w_{U_{\infty}}\right)^{2}+\left(\frac{\partial C_{D}}{\partial c} w_{c}\right)^{2}+\left(\frac{\partial C_{D}}{\partial L} w_{L}\right)^{2}\right\}^{1 / 2}
$$

\section{Particle Image Velocimetry Measurement}

As for the force measurements described above, the low-turbulence water tunnel at University of Michigan was employed. The cross section is $61 \mathrm{~cm}$ height by $61 \mathrm{~cm}$ wide; the turbulence intensity is approximately $1 \%$ and the free stream velocity ranges from $5 \mathrm{~cm} / \mathrm{s}$ to $40 \mathrm{~cm} / \mathrm{s}$.

To determine the flow evolution two dimensional Particle Image Velocimetry (PIV) was used. The PIV system includes a double-pulsed Nd-YAG laser (Spectra Physics PIV 300), light sheet formation optics, a dual frame digital camera (Cooke Corp. PCO.4000), a computer image acquisition system and control electronics. The laser sheet has a thickness of approximately $1 \mathrm{~mm}$ and was located at about the mid-span plane of the model. The water channel was seeded with $5 \mu \mathrm{m}$ diameter Titanium Dioxide particles (Sigma-Aldrich). The cameras were installed underneath the water channel test section and were equipped with Nikon 105-mm Micro-Nikkor lenses to produce a magnification of approximately 15.2 pixels $/ \mathrm{mm}$. 100 image pairs were recorded at selected phases of the motion. With this magnification, the exposure time was adjusted to produce a nominal particle displacement of 2 pixels in order to minimize the effect of spanwise flow in the measurements. The PCO.4000 camera frame size 4008 by 2672 pixels gives a field of view 263 by $175 \mathrm{~mm}$ in the flow. The PIV system was used to measure the free stream velocity which was found to be $7.04 \mathrm{~cm} / \mathrm{s}$ with standard deviation of $0.66 \mathrm{~cm} / \mathrm{s}$ and the accuracy about $3.15 \mathrm{e}-004$ $\mathrm{cm} / \mathrm{s}$.

PIV measurements were conducted for $\alpha_{\mathrm{m}}$ equal to $45^{\circ}$ only. PIV images were taken at phases shown as vertical lines in Figure 4. The corresponding angles during pitch ramp and return are approximately $0^{\circ}, 3^{\circ}, 13^{\circ}, 22^{\circ}, 31^{\circ}, 41^{\circ}$, and $45^{\circ}$, which are $0.47 \mathrm{sec}$ apart. During the holding, the first two snapshots are 0.28 seconds apart; the rest are 0.56 seconds apart.

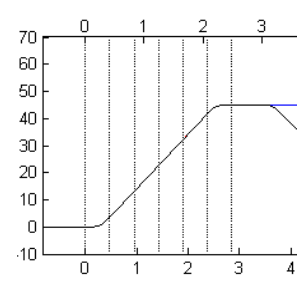

(a)

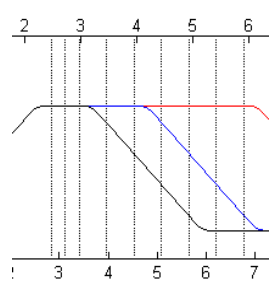

(b)

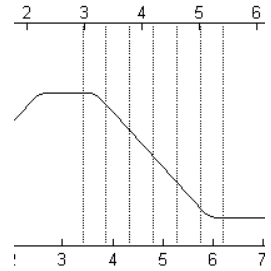

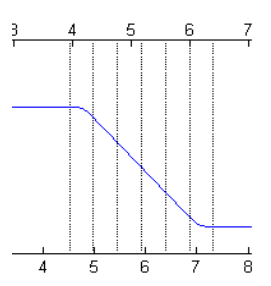

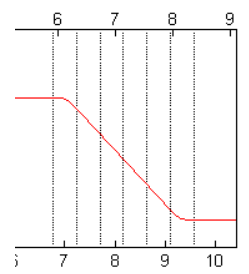

(c)

Figure 4. Snapshots for (a) pitching ramp motion; (b) the holding; (c) the pitching return, $h=0.5,1,2$ from the left to the right for $\alpha_{\mathrm{m}}=45^{\circ}$

\section{Results and Discussion}

Force coefficient time history at $\alpha_{\mathrm{m}}$ of $33^{\circ}, 45^{\circ}$ and $57^{\circ}$ are presented in Figures 5-7, respectively. The right ordinate of the plots is the angle of attack, and the left ordinate is the corresponding force coefficient. The convective time is shown in the top abscissa, and the bottom abscissa gives the physical time. There are four spikes in all the lift force traces which coincide with the acceleration of the wing. Clearly, the forces are better described based on the different phases of the wing motion: the pitch ramp phase, the holding phase, and the return phase.

All the PIV measurement results are presented in phase averaged vorticity contours and streamlines. The RGB color schemes are employed, and in all cases the normalized contour levels are -0.08 to +0.08 , with contour values in the range -0.01 to +0.01 not shown for clarity. Data in the shadow region of the light sheet caused by the model are masked and shown as a white region. Model glare at the surface may cause unreliability of the velocity data adjacent to the wing surface. Synchronization between motion and camera was performed using external trigged signals; and found to be within $0.4^{\circ}$. The free stream velocity measured with the PIV is $7.04 \mathrm{~cm} / \mathrm{s}$ with a standard deviation of $0.66 \mathrm{~cm} / \mathrm{s}$. 

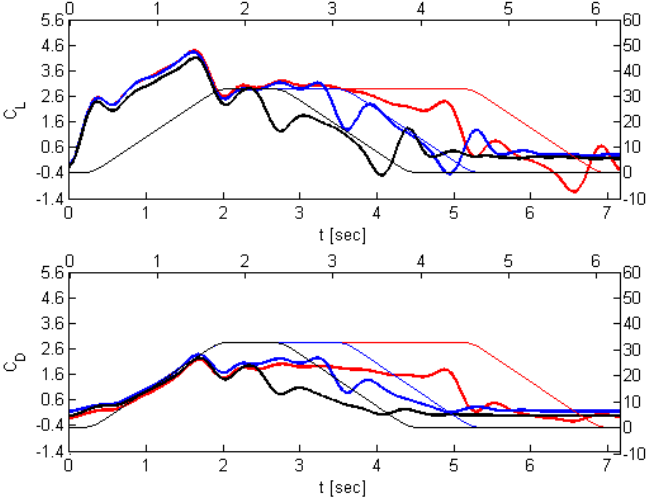

(a)
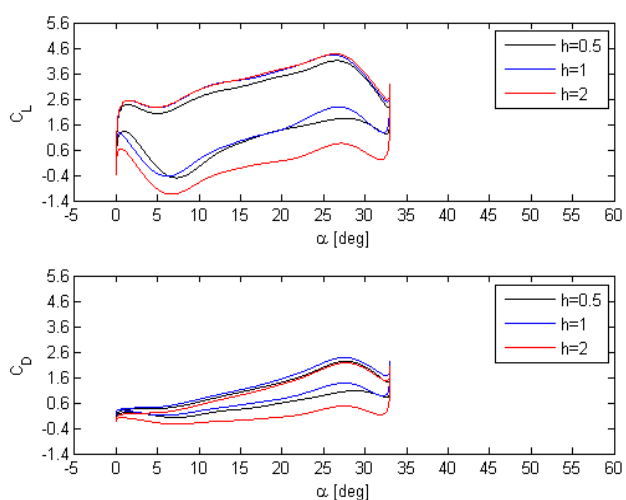

(b)

Figure 5. Force coefficient time and angular history comparison of various holding parameters at $\alpha_{\mathrm{m}}=33^{\circ}$
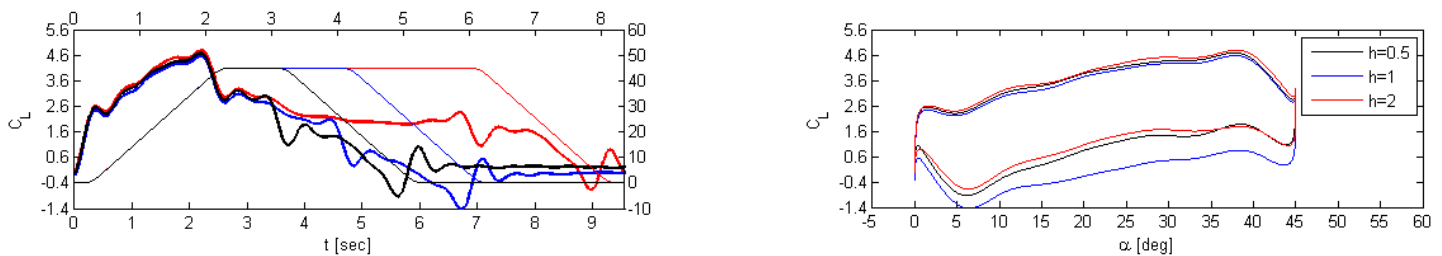

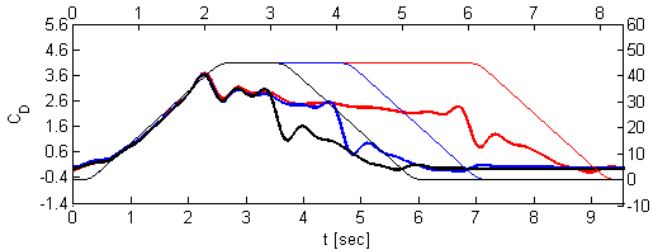

(a)

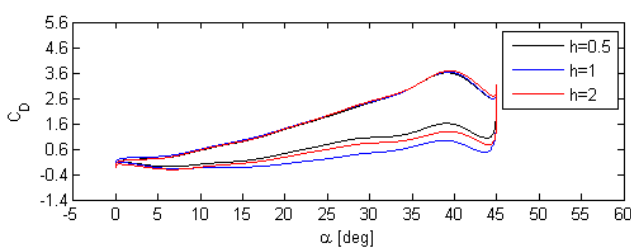

(b)

Figure 6. Force coefficient time and angular history comparison various holding parameters at $\alpha_{\mathrm{m}}=45^{\circ}$
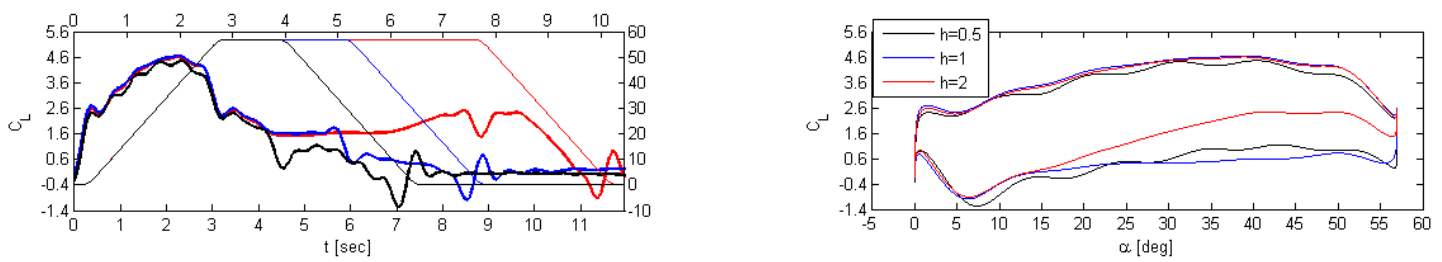

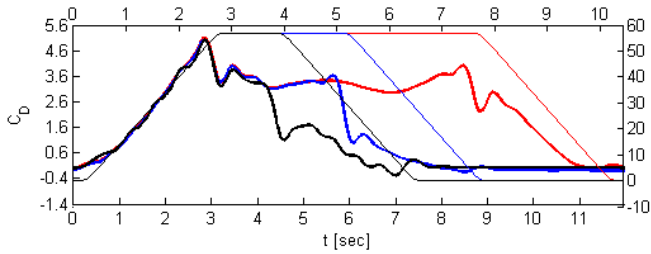

(a)

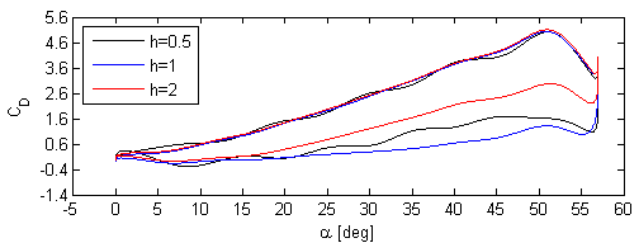

(b)

Figure 7. Force coefficient time and angular history comparison of various holding parameters at $\alpha_{\mathrm{m}}=57^{\circ}$

\section{A. The Pitch Ramp Phase}

Figure 8 shows the force coefficient plotted vs. pitch angle during the linear pitch ramp part of the motion, which is the region between $t s 1$ and $t s 4$ in Figure 1(a). The vertical lines indicate the phases at which PIV measurements were obtained for $\alpha_{m}$ equal to $45^{\circ}$ only. The black, blue, and red curves represent $\alpha_{m}$ of $33^{\circ}, 45^{\circ}$, and $57^{\circ}$. The lift increases rapidly due to non-circulatory effects ${ }^{1}$ at the start of the motion, followed by a slower increase as the pitch 
angle increases, and then saturation of the lift coefficient at about $35^{\circ}-40^{\circ}$ and a decrease in lift after saturation. When the motion enters the second transition the lift decreases rapidly, much faster than the decrease after saturation. The first transition does not impact drag, and the drag increases with maximum angle before entering the second transition. The force during the pitch ramp motion is independent of the maximum angle of attach and holding duration. The dotted line in Figure 8 is $2 \pi \alpha$ which is the result for thin airfoil theory. This curve intersects the present force measurement results at about $40^{\circ}$. For pitch angles less than $40^{\circ}$ the lift force produced by the unsteady motion is significantly larger than the value predicted by steady theory.
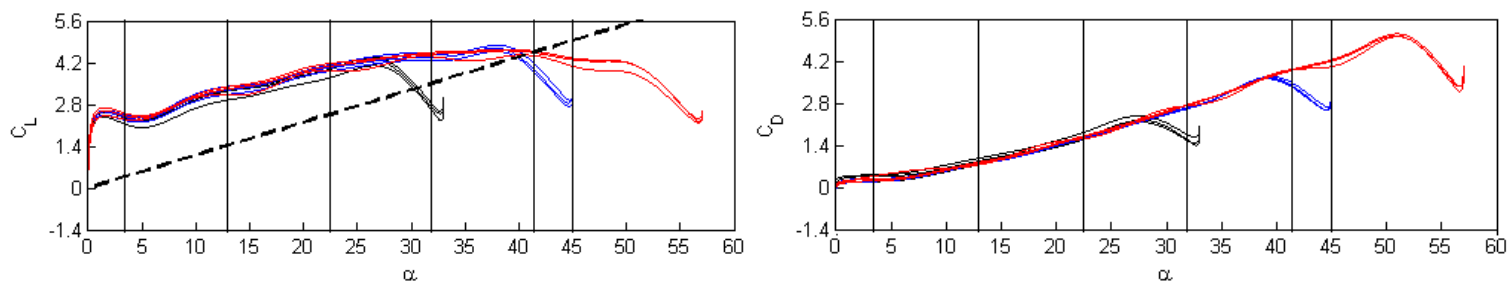

Figure 8. Force coefficient angular history from various $\alpha_{m}$ during the pitch ramp

For the present results with $\mathrm{k}=0.2, \alpha_{\mathrm{m}}=45^{\circ}, \mathrm{Re}=5000$, and leading edge pivot point, the maximum lift coefficient is approximately 4.6, which is significantly higher than the 3.8 value reported by $\mathrm{Ol}$ et al ${ }^{1} \mathrm{Lian}_{\text {and } \mathrm{Ol}^{7}}$ investigated the effects of blockage using numerical simulations and found maximum lift values as high as 5.5 when the channel walls are located one chord away from the model. The maximum value decreases to approximately 4 when the test section walls are located 10 chords lengths away from the model. The value for test section walls located 4 chords away from the model, which is the distance for the present experiments, is 4.4 consistent with the present results. Force measurement uncertainty results are shown in Figure 9 as a function of angle of attack. Highest measurement uncertainty is found at small pitch angles during the first transient. These results show that in all cases the measurement uncertainty is within $3 \%$ of the maximum values.
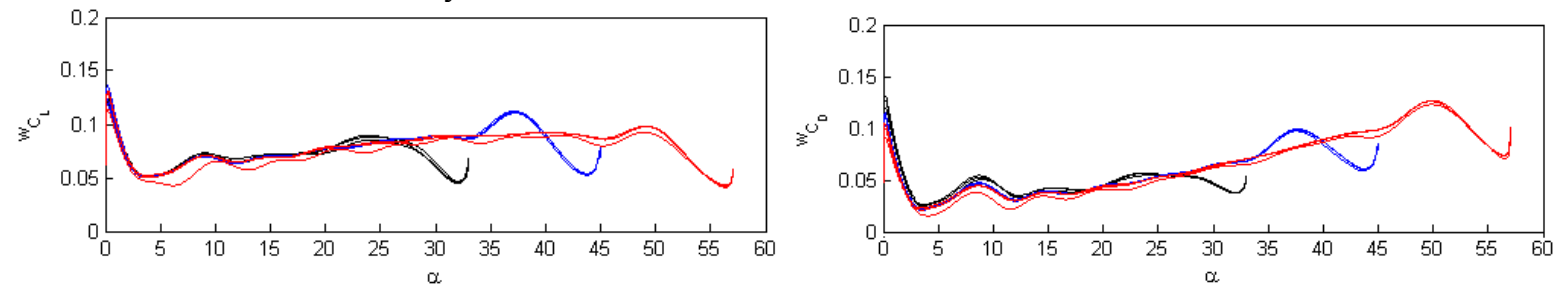

Figure 9. Uncertainty of the force coefficients during the pitch ramp

Figure 10 shows the flow development during the pitch ramp phase of the motion for $\alpha_{m}$ equal to $45^{\circ}$. The images are spaced 0.474 seconds, corresponding to 0.414 convective times. The corresponding pitch angle does not vary linearly with time due to smoothing at the transitions. Initially, clockwise vorticity is observed on the upper surface of the wings. As pitching angle increases, a counterclockwise rotating starting vortex quickly forms in the wake. Formation of leading edge vortex is slow. There is no apparent LEV vortex formed at the first three snapshots. As pitching angle increases, the attached vorticity on the upper surface increases at the leading edge, and a LEV begins to form at $\alpha=22.4^{\circ}$. The LEV develops quickly between pitching angles of $22.4^{\circ}$ to $31.9^{\circ}$. This LEV grows in size after $41.4^{\circ}$. These results are similar to earlier results reported by Baik ${ }^{3}$. In his case for pivot point at quarter chord, the LEV forms at about $36^{\circ}$ which in reasonable agreement with the present observations for pivot point at the leading edge. When the LEV appears is formed counterclockwise vorticity is also observed on the upper surface of the wing which roles up into a secondary vortex. Comparison with the force measurement indicates that lift saturation occurs during the development of the secondary vortex on the surface of the plate. At the trailing edge a starting vortex with counterclockwise vorticity is formed at the initiation of the motion. A shear layer develops which rolls up into multiple smaller vortices and the motion continues possible due to Kelvin-Helmholtz instability. 

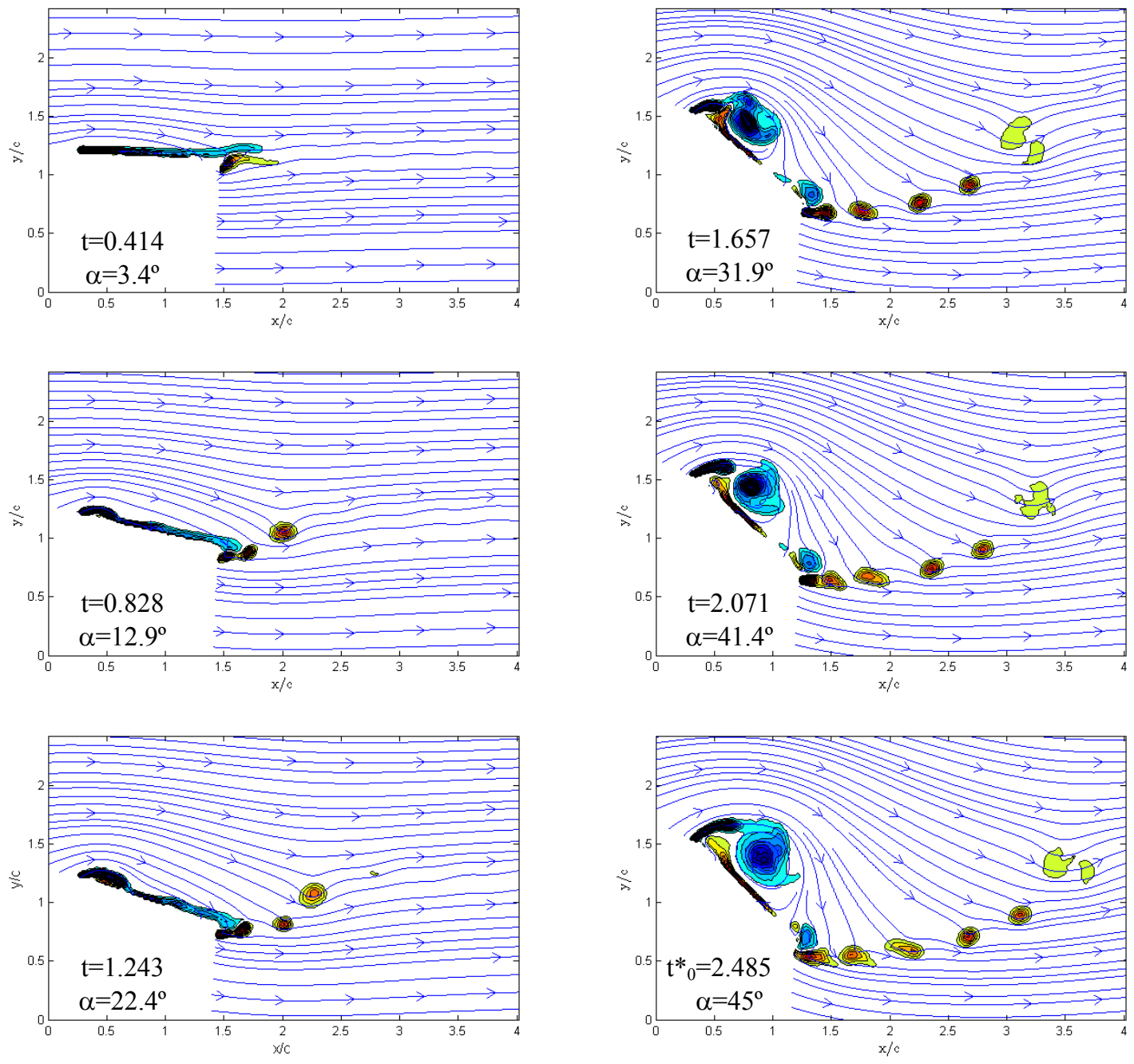

Figure 10. Flow topology for linear pitching ramp motion at $\alpha_{\mathrm{m}}=45^{\circ}, \mathrm{k}=0.2 . \mathrm{t}^{*}{ }_{0}$ indicates the holding starts

\section{B. The Holding Phase}

Figure 11 shows the force coefficient as a function of convective time during the hold part of the motion for different $\alpha_{\mathrm{m}}$ as noted in the caption. The hold phase corresponds to the region between $t s 4$ to $t s 5$ in Figure 1(a). The black, blue and red curves indicate the holding parameters of $0.5,1$, and 2 . The force coefficients histories have different behavior depending on maximum pitch angle. For $\alpha_{\mathrm{m}}$ equal to $33^{\circ}$, the LEV is not fully formed at the start of the hold phase, and the maximum pitch angle is reached before lift saturation. The force measurement results shown in Figure 11(a) suggest that the flow undergoes further development of LEV with an increase in the lift coefficient. As hold time continues the lift coefficient decreases after approximately one convective. The reduction in lift is faster than in drag. For the case $\alpha_{\mathrm{m}}=45^{\circ}$, the LEV is well formed at the beginning of the hold phase, and the lift reaches a maximum (saturate) just before the maximum pitch angle is reached. As shown in Figure 11(b), lift and drag coefficients decrease within the first convective time, while the drag coefficient decreases slowly. For $\alpha_{m}$ equal to $57^{\circ}$, the attached LEV is well-formed and larger than for $\alpha_{m}=45^{\circ}$, lift saturates before the maximum pitch angle is reached. The lift decreasing during one convective time, and increases after 3 convective times as shown in Figure 11(c). The drag coefficient shows an oscillation within 1 to 3 convective times. 

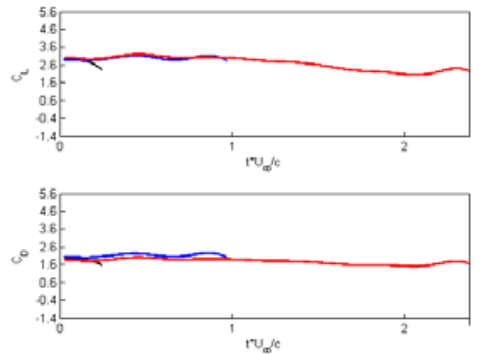

(a)
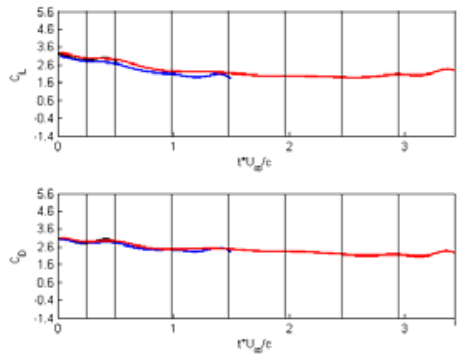

(b)
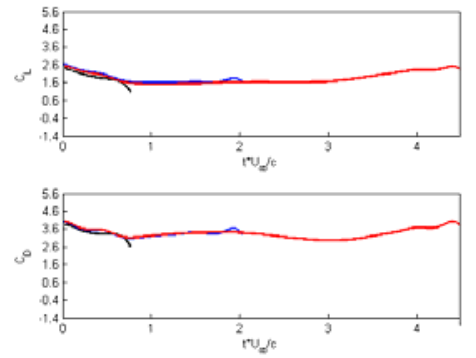

(c)

Figure 11. Force coefficient time history during the holding (a) $\alpha_{m}=33^{\circ}$ (b) $\alpha_{m}=45^{\circ}$ (c) $\alpha_{m}=57^{\circ}$

The PIV measurement results for $\alpha_{\mathrm{m}}=45^{\circ}$ are shown in Figure 12. The first two images were obtained 0.28 seconds apart, and the others 0.56 seconds apart. The reference time $t^{*}{ }_{0}$ represents the nondimensional time right at the start of the hold phase of the motion. The flow topology at $t^{*}{ }_{0}$ is shown in Figure 10 . At the start of the hold phase there is a well-developed LEV on the suction side of the plate. A secondary vortex is also observed produced by the LEV-induced velocity field at the surface of the plate. At the trailing edge and near wake, vorticity shed at the trailing edge results in a starting vortex and a sequence of smaller vortices produced by Kelvin-Helmholtz instability in the separated shear layer. As the flow evolves during the hold phase of the motion, a new trailing edge vortex (TEV) forms (see flow field at 1.469 convective times) as the LEV detaches. The TEV vortex also detaches at approximately 2.5 convective times and a new LEV forms, indicating the onset of vortex shedding. However steady vortex shedding never develops because the return phase stops the process. In Figure 12, the flow fields at the start of the return phase for the different hold parameters are highlighted. For the short hold parameter, 0.5 which corresponds to 0.49 convective time, the LEV vortex is fully developed and a small TEV vortex is observed at the trailing edge. For the intermediate hold parameter, 1 which corresponds to 1.469 convective time, the LEV is detached and the TEV attached and large. For the large hold parameter, 2 which corresponds to 3.428 convective times, the LEV from the previous shedding cycle has advected downstream, the TEV is detached and a new LEV is formed at the leading edge. These differences in flow topology at the start of the return phase of the motion can be expected to impact the force history and flow evolution during the return phase. 

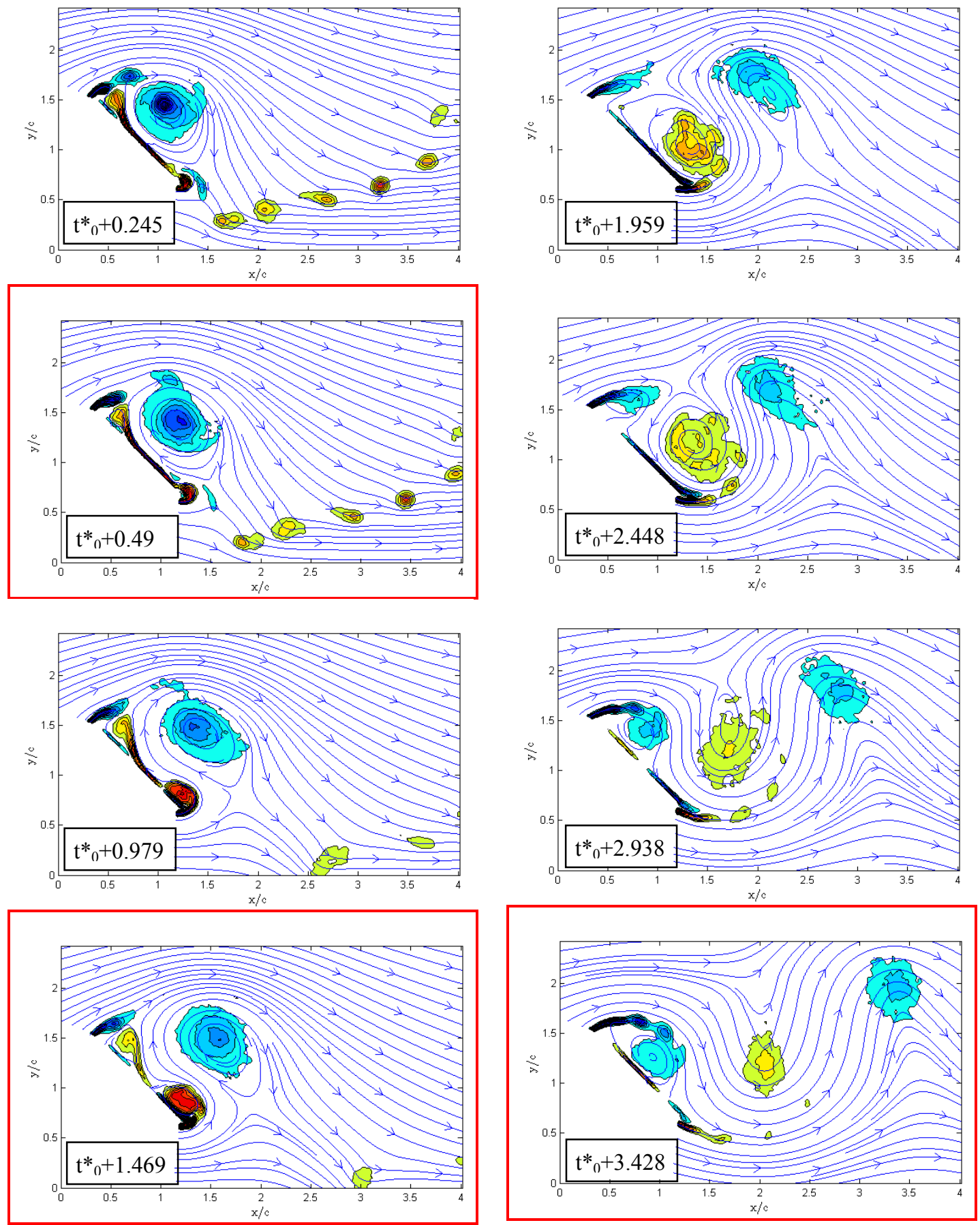

Figure 12. Flow topology during the hold phase for $\alpha_{\mathrm{m}}=45^{\circ}, \mathrm{k}=\mathbf{0 . 2}$. Red box indicates the pitch return starts for $h=0.5,1,2$ in sequence.

\section{The Return Phase}

Now we consider the return motion of the wing for the various holding times at the different maximum angle of attack, $\alpha_{\mathrm{m}}$. The force coefficient time histories are shown in Figure 13. The black, blue and red curves indicate the hold parameter values of $0.5,1$, and 2 respectively. The pitch angle history is also plotted in Figure 13 (right vertical axis) and is the same for all cases. The return phase corresponds to time range from $t s 5$ to $t s 8$ shown in Figure 1(a), 
where the smoothing transitions are included at the start and end of the motion. Two lift force spikes are observed corresponding to the acceleration of the plate and are attributed to non-circulatory effects. Outside the noncirculatory spikes, the forces decrease with time and pitch angle. Different hold parameter yield different force time histories. The reduction in lift coefficient is more pronounced that the reduction in drag coefficient. For $\alpha_{\mathrm{m}}$ equal to $33^{\circ}$, similar force time histories are found for $h=0.5$ and $h=1$, and lower force for $h=2$. For $\alpha_{m}$ equal to $45^{\circ}$, similar force time histories are found for $h=0.5$ and $h=2$, and lower force for $h=1$. For $\alpha_{m}$ equal to $57^{\circ}$, similar force time histories are found for $h=0.5$ and $h=1$, and higher force for $h=2$.
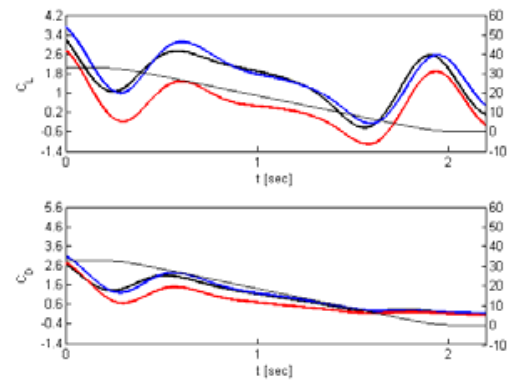

(a)
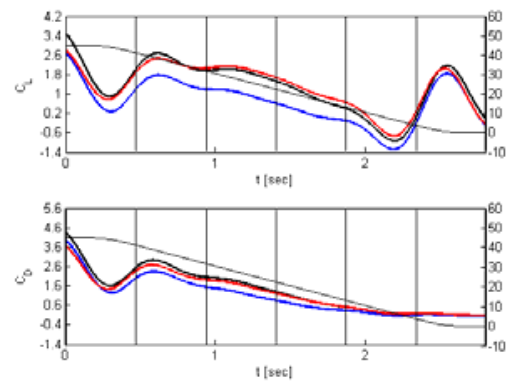

(b)

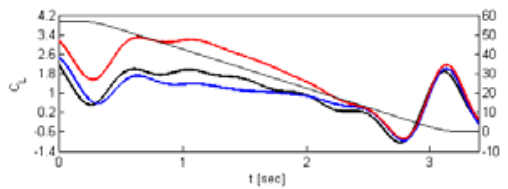

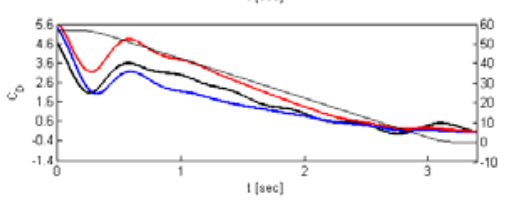

(c)

Figure 13. Force coefficient time history during the return motion (a) $\alpha_{\mathrm{m}}=33^{\circ}$ (b) $\alpha_{\mathrm{m}}=45^{\circ}$ (c) $\alpha_{\mathrm{m}}=57^{\circ}$, black curves: $h=0.5$, blue curves: $h=1$; red curves: $h=2$.

These force measurement results show that the force history and flow evolution history depend on both maximum angle and hold parameter. The PIV measurements conducted for $\alpha_{\mathrm{m}}$ equal to $45^{\circ}$ and shown in Figure 14 provide useful insight on these similarities and differences. In Figure 14, $t_{d 1}, t_{d 2}$ and $t_{d 3}$ represent the time when the return motion starts for $\mathrm{h}=0.5,1$, and 2, respectively. For each case, the images were taken at the same phases, which are 0.467 seconds apart. There are 7 images taken to capture the flow evolution for the different hold parameter. For $\mathrm{h}=0.5$, a secondary vortex and a TEV are developing in the first two snapshots. As the pitch angle decreases, the secondary vortex dissipates and is convected to merge with the TEV after 1.63 convective times. The upper surface is then covered by the clockwise vorticity as the pitch angle return to zero, the starting position. For $h$ $=1$, a secondary vortex and a TEV are present at the beginning of the return phase. These vortices detach and are advected into the wake where they dissipate as the pitch angle decreases. For $h=2$, a LEV with weaker vorticity is observed at the start of the return motion as well as the LEV and TEV from the previous shedding in the near wake. As the pitch angle decrease the LEV dissipates before detaching and the wake vortices dissipate.

The force measurements for $\mathrm{h}=0.5$ and 2 have about the same history during the return phase whereas the case for $\mathrm{h}=1$ have lower forces. The PIV measurement shows an LEV vortex and no TEV for $\mathrm{h}=0.5$ and 2 . These observations suggest that these two cases correspond to the same phase in the vortex shedding cycle at the start of the return phase. While for $\mathrm{h}=1$ the flow topology at the start of the return phase consists of detach LEV and a strong TEV which correspond to intermediate state in the vortex shedding cycle which results in reduced force during the return phase. Assuming that the time delay between $h=0.5$ and $h=2$ defines the period of vortex shedding at 45 degrees the estimated value of the Strouhal number would be,

$$
\mathrm{St}=\frac{\mathrm{c} \sin \alpha_{\mathrm{m}}}{\mathrm{TU}_{\infty}}=0.22
$$




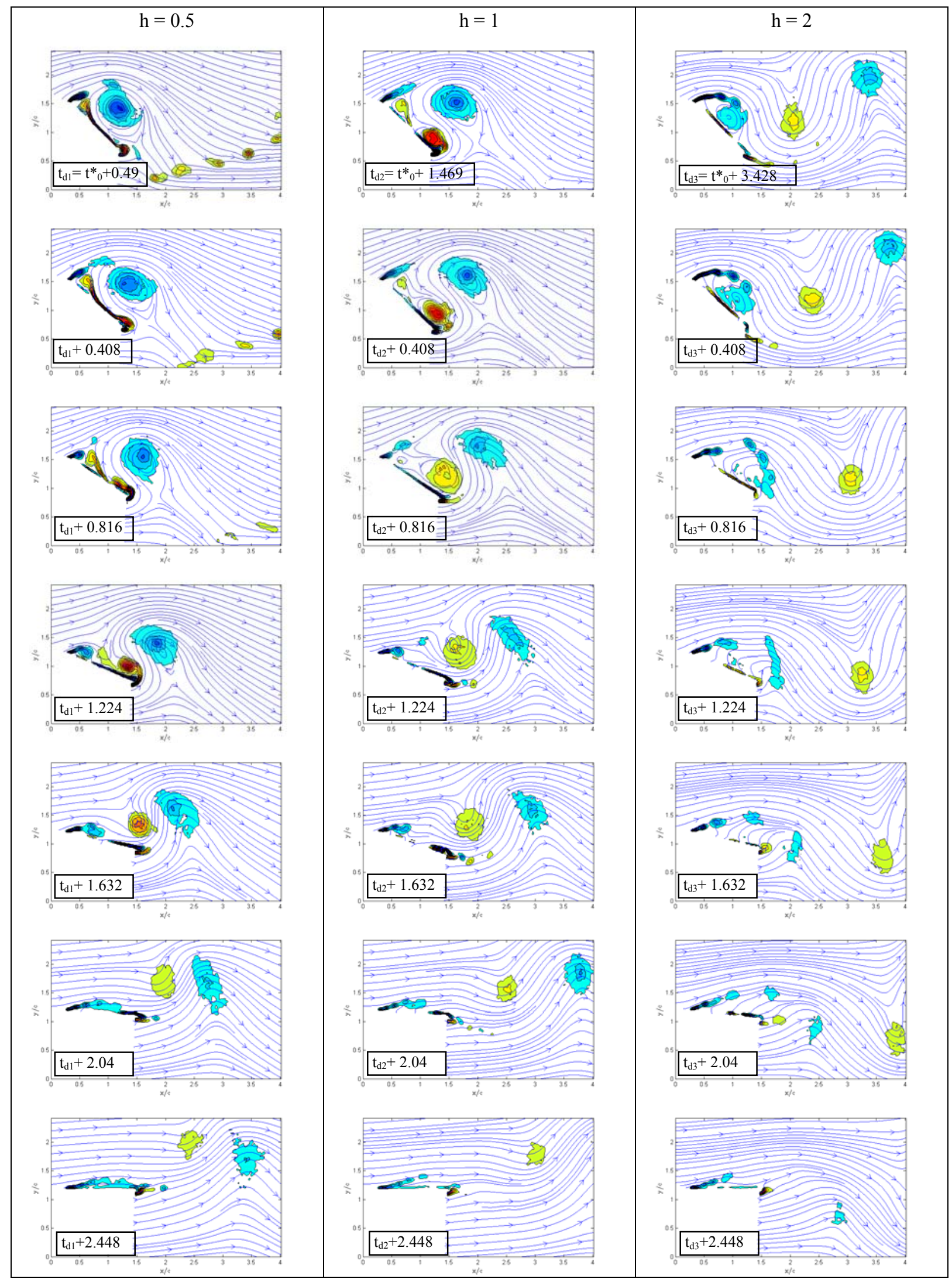

Figure 14. Flow topology for pitching return motion at $\alpha_{\mathrm{m}}=45^{\circ}, \mathrm{k}=0.2$.

American Institute of Aeronautics and Astronautics 


\section{Conclusion}

In this study, experiments were conducted to determine the force time history and flow evolution history for a pitch ramp-hold-return kinematics at a Reynolds number of 5000 and reduced frequency of 0.2 . Direct force measurement and PIV measurement results are reported. The pitch ramp, hold, and return phases of the motion have different characteristics depending on maximum pitch angle and hold time. The force time history during the pitch ramp phase is in good agreement with previous work. Four force spikes are found corresponding to the acceleration of the wing, which are due to non-circulatory effects. Increasing the maximum pitch angle increases drag significantly more than lift during the pitch up phase. During the hold phase, the evolution of aerodynamic parameters is a function of time and maximum pitch angle but independent of hold duration. During the hold phase, LEV detachment and the development of a secondary vortex and a trailing edge vortex results in decrease of lift. During the hold phase, for long holding duration incipient vortex shedding is observed with Strouhal number of 0.22 for $\alpha_{\mathrm{m}}=45^{\circ}$. The force and flow evolution during the return phase depend on the hold duration and maximum pitch angle. These are attributed to the different vortex shedding phase at the end of the hold phase.

\section{Acknowledgement}

The work was sponsored in part by the Air Force Office of Scientific Research's Multidisciplinary University Research Initiative (MURI) on Biologically-Inspired Anisotropic Flexible Wing for Optimal Flapping Flight and by the Michigan/AFRL (Air Force Research Laboratory) Collaborative Center in Aeronautical Sciences.

\section{References}

${ }^{1}$ Ol, M. V., Altman, A., Eldredge, J. D., Garmann, D. J., Lian, Y., Résumé of the AIAA FDTC Low Reynolds Number Discussion Group's Canonical Cases, 48th AIAA Aerospace Sciences Meeting, Orlando, FL, AIAA 2010-1085.

${ }^{2}$ Granlund, K., Ol, M., Garmann, D., Visbal, M., Bernal, L., "Experiments and Computations on Abstractions of Perching", 28th AIAA Applied Aerodynamics Conference, Chicago, IL, AIAA 2010-4943.

${ }^{3}$ Baik, Y. S., Aono, A. Rausch, J. M., Bernal, L. P., Shyy, W., "Experimental Study of a Rapidly Pitched Flat Plate at Low Reynolds Number",", 40th AIAA Fluid Dynamics Conference, AIAA 2010-4462.

${ }^{4}$ Ol, M. V., Eldredge, J. D., Wang, C., "High-Amplitude Pitch of a Flat Plate: an Abstraction of Perching and Flapping", International Journal of Micro Air Vehicles, Vol. 1, No. 3, Sep. 2009.

${ }^{5}$ Eldredge, J. D. and Wang, C., “ A Computational Study of a Canonical Pitch-up, Pitch-down Wing Maneuver”, 39th AIAA Fluid Dynamics Conference, AIAA 2009-3687.

${ }^{6}$ Garmann, D. J. and Visbal, M. R., "High-Fidelity Simulations of Transitional Flow Over Pitching Airfoils", 39th AIAA Fluid Dynamics Conference, AIAA 2009-3693.

${ }^{7}$ Lian, Y. and OL, M. V., "Computation and Experiments on a Low Aspect Ratio Pitching Flat Plate", 48th AIAA Aerospace Sciences Meeting Including the New Horizons Forum and Aerospace Exposition, AIAA paper 2010-0385. 